\title{
ON FOURIER SERIES WITH RESTRICTED COEFFICIENTS*
}

\author{
OTTO SZÁSZ
}

1. Introduction. Consider a real-valued function $f(x)$, periodic with period $2 \pi$ and Lebesgue integrable. Let

$$
f(x) \sim \frac{a_{0}}{2}+\sum_{1}^{\infty}\left(a_{\nu} \cos \nu x+b_{\nu} \sin \nu x\right)
$$

be its Fourier series, and let $s_{0}=a_{0} / 2$,

$$
\begin{aligned}
s_{n}(f ; x)=s_{n}=\frac{a_{0}}{2}+\sum_{1}^{n}\left(a_{\nu} \cos \nu x+b_{\nu}\right. & \sin \nu x), \\
& n=1,2,3, \cdots,
\end{aligned}
$$

be its partial sums. We shall mainly restrict ourselves to series satisfying the conditions

$$
n a_{n} \geqq-p, \quad n b_{n} \geqq-p, \quad \text { for } \quad n=1,2,3, \cdots,
$$

where $p \geqq 0$. We shall in particular consider the following problem:

Suppose

$$
-\mu \leqq f(x) \leqq \mu \quad \text { in } \quad-\pi<x<\pi ;
$$

then what is the best upper bound $C_{n}(\mu, p)$ for the partial sums $\left|s_{n}(f ; x)\right| \leqq C_{n}(\mu, p),(n \geqq 1)$, under the assumption (1.3)?

It is known that the sequence $\left\{C_{n}\right\}$ is bounded (cf. Szász [5], [6]); $\dagger$ hence l.u.b. $C_{n}(\mu, p)=C(\mu, p)$ is finite. For $p=0$ the author [9] proved recently that

$$
C(\mu, 0)<(2+4 / \pi) \mu<3.3 \mu ;
$$

for $p>0$ the sharpest estimates so far were given by Fekete [2] using a device of Paley and Fejér [1]. Fekete proved that

$$
C(\mu, p)<5 \mu+6 p
$$

and also that

$$
C(\mu, p)<5 \mu+8(\mu p)^{1 / 2}
$$

* Presented to the Society, April 9, 1938.

$\dagger$ See the list of references at the end of this paper. 
Note that for any $\rho>0$, we have $s_{n}(\rho f ; x)=\rho s_{n}(f ; x)$, which gives the relation $\rho C_{n}(\mu, p)=C_{n}(\rho \mu, \rho p),(p \geqq 0, \rho>0)$, and in particular

$$
C_{n}(\mu, p)=\mu C_{n}(1, p / \mu) \text {. }
$$

Hence in the discussion we may put $\mu=1$; that is, $|f(x)| \leqq 1$. Using a similar tool as in [9] we shall improve upon the known results. We shall get sharper estimates for the $k$ th partial sum assuming (1.3) only for $n=1,2, \cdots, 2 k-1$.

2. Certain identities and inequalities. Given an infinite series $\sum_{0}^{\infty} u_{\nu}$ let $\sum_{0}^{n} u_{\nu}=U_{n}, \sum_{0}^{n-1} U_{\nu}=U_{n}^{(1)}=\sum_{0}^{n}(n-\nu) u_{\nu},(n=0,1,2, \cdots)$; we then have

$$
\begin{aligned}
\frac{1}{2 n} U_{2 n}^{(1)}-\frac{1}{n} U_{n}^{(1)} & =\frac{1}{2 n}\left\{\sum_{0}^{2 n}(2 n-\nu) u_{\nu}-2 \sum_{0}^{n}(n-\nu) u_{u}\right\} \\
& =\frac{1}{2 n}\left\{\sum_{0}^{n} \nu u_{\nu}+\sum_{n+1}^{2 n}(2 n-\nu) u_{\nu}\right\},
\end{aligned}
$$

for $n=1,2, \cdots$, and $U_{n}^{(1)}=n \sum_{0}^{n} u_{\nu}-\sum_{0}^{n} \nu u_{\nu}=n U_{n}-\sum_{0}^{n} \nu u_{\nu}$, or

$$
U_{n}=\frac{1}{n} U_{n}^{(1)}+\frac{1}{n} \sum_{1}^{n} \nu u_{\nu}, \quad n=1,2, \cdots .
$$

We get similarly

$$
U_{n}=\frac{1}{2 n} U_{2 n}^{(1)}+\left(\frac{1}{2 n} U_{2 n}^{(1)}-\frac{1}{n} U_{n}^{(1)}\right)-\frac{1}{n} \sum_{n+1}^{2 n}(2 n-\nu) u_{\nu}
$$

for $n=1,2, \cdots$, and $([4]$, p. 186)

$$
\begin{array}{r}
U_{n}=\frac{U_{n+\kappa}^{(1)}}{n+\kappa}+\frac{n}{\kappa}\left(\frac{U_{n+\kappa}^{(i)}}{n+\kappa}-\frac{U_{n}^{(1)}}{n}\right)-\frac{1}{\kappa} \sum_{\nu=n+1}^{n+\kappa}(n-\nu+\kappa) u_{\nu}, \\
\kappa \geqq 1, n \geqq 1 .
\end{array}
$$

This reduces to (2.3) for $\kappa=n$, whereas for $\kappa=1$ it yields

$$
U_{n}=\frac{1}{n+1} U_{n+1}^{(1)}+\frac{1}{n+1} \sum_{1}^{n} \nu u_{\nu}
$$

We are using in addition the fact that the assumption

$$
-1 \leqq f(x) \leqq 1 \text { for }-\pi<x<\pi
$$

involves

$$
\left|\sum_{0}^{n-1} s_{p}(x)\right| \leqq n, \quad n=1,2, \cdots
$$


which is the classical result of Fejér, and (compare [9]) the relations

$$
\begin{aligned}
& \left|\sigma_{2 n}(x)-\sigma_{n}(x)\right| \leqq 2 / \pi, \\
& \left|\bar{\sigma}_{2 n}(x)-\bar{\sigma}_{n}(x)\right| \leqq 2 / \pi, \quad n=1,2, \cdots,
\end{aligned}
$$

where $\sigma_{n}=(1 / n) \sum_{0}^{n-1} s_{\nu}$, and $\bar{\sigma}_{n}$ is the corresponding mean for the conjugate series. In view of (2.1) the last inequalities can be written as

$$
\begin{aligned}
\mid \sum_{1}^{n} \nu\left(a_{\nu} \cos \nu x\right. & \left.+b_{\nu} \sin \nu x\right) \\
& +\sum_{n+1}^{2 n}(2 n-\nu)\left(a_{\nu} \cos \nu x+b_{\nu} \sin \nu x\right) \mid \leqq \frac{4}{\pi} n \\
\mid \sum_{1}^{n} \nu\left(b_{\nu} \cos \nu x\right. & \left.-a_{\nu} \sin \nu x\right) \\
& +\sum_{n+1}^{2 n}(2 n-\nu)\left(b_{\nu} \cos \nu x-a_{\nu} \sin \nu x\right) \mid \leqq \frac{4}{\pi} n
\end{aligned}
$$

We shall use also the following more general inequalities:

$$
\begin{aligned}
& \mid \sum_{\nu=1}^{n} \nu\left\{a_{\nu+\lambda-1} \cos (\nu+\lambda-1) x+b_{\nu+\lambda-1} \sin (\nu+\lambda-1) x\right\} \\
& \quad+\sum_{\nu=n+1}^{2 n}(2 n-\nu)\left\{a_{\nu+\lambda-1} \cos (\nu+\lambda-1) x+b_{\nu+\lambda-1} \sin (\nu+\lambda-1) x\right\} \mid \leqq \frac{4}{\pi} n, \\
& \mid \sum_{\nu=1}^{n} \nu\left\{b_{\nu+\lambda-1} \cos (\nu+\lambda-1) x-a_{\nu+\lambda-1} \sin (\nu+\lambda-1) x\right\} \\
& \quad+\sum_{\nu=n+1}^{2 n}(2 n-\nu)\left\{b_{\nu+\lambda-1} \cos (\nu+\lambda-1) x-a_{\nu+\lambda-1} \sin (\nu+\lambda-1) x\right\} \mid \leqq \frac{4}{\pi} n, \\
& \quad \lambda \geqq 1-n / 2 .
\end{aligned}
$$

3. Two auxiliary theorems. We prove the following lemma:

Lemma 1. The sequence $\rho_{n}=n^{-1} \sum_{n+1}^{2 n}(2 n-\nu) / \nu,(n=1,2,3, \cdots)$, is monotonically increasing, and $\rho_{n} \uparrow 2 \log 2-1=0.38629 \ldots$.

For the proof note that

$$
\begin{aligned}
\rho_{n+1}-\rho_{n} & =2\left(\sum_{n+2}^{2 n+2} \frac{1}{\nu}-\sum_{n+1}^{2 n} \frac{1}{\nu}\right)=2\left(\frac{1}{2 n+1}+\frac{1}{2 n+2}-\frac{1}{n+1}\right) \\
& =\frac{2}{2 n+1}-\frac{1}{n+1}>0 ;
\end{aligned}
$$

furthermore 


$$
\rho_{n}=\frac{1}{n} \sum_{n+1}^{2 n} \frac{2-\nu / n}{\nu / n} \rightarrow \int_{1}^{2} \frac{2-x}{x} d x=2 \log 2-1 .
$$

LEMma 2. The sequence $\tau_{n}=n^{-1} \sum_{n}^{2 n-1}(\nu-n) / \nu,(n=1,2,3, \cdots)$, is monotonically increasing, and

$$
\tau_{n} \uparrow 1-\log 2=0.30685 \cdots \text {. }
$$

Indeed

$\tau_{n+1}-\tau_{n}=\sum_{n}^{2 n-1} \frac{1}{\nu}-\sum_{n+1}^{2 n+1} \frac{1}{\nu}=\frac{1}{n}-\frac{1}{2 n}-\frac{1}{2 n+1}=\frac{1}{2 n}-\frac{1}{2 n+1}>0$ and

$$
\tau_{n}=\frac{1}{n} \sum_{n}^{2 n-1} \frac{\nu / n-1}{\nu / n} \rightarrow \int_{1}^{2} \frac{x-1}{x} d x=1-\log 2 .
$$

4. Estimates for $\sum \nu\left|a_{\nu}\right|, \sum \nu\left|b_{\nu}\right|$, and related sums. In view of (2.1) the inequalities (2.8) can be written as

$$
\left|\sum_{1}^{n} \nu u_{\nu}+\sum_{n+1}^{2 n}(2 n-\nu) u_{\nu}\right| \leqq \frac{4}{\pi} n, \quad n=1,2, \cdots,
$$

where $u_{\nu}$ is $a_{\nu} \cos \nu x+b_{\nu} \sin \nu x$ or $b_{\nu} \cos \nu x-a_{\nu} \sin \nu x$, respectively. In particular, for $x=0$, we have $\sum_{1}^{n} \nu a_{\nu}+\sum_{n+1}^{2 n+1}(2 n-\nu) a_{\nu} \leqq 4 n / \pi,(n \geqq 2)$, and $\left|a_{1}\right| \leqq 4 / \pi ;$ hence

$$
\begin{aligned}
& \sum_{1}^{n}\left(\nu a_{\nu}+p\right)+\sum_{n+1}^{2 n-1}(n-\nu)\left(a_{\nu}+\frac{p}{\nu}\right) \\
& \leqq n\left(\frac{4}{\pi}+p\right)+p \sum_{n+1}^{2 n-1} \frac{2 n-\nu}{\nu}, \quad n \geqq 2 .
\end{aligned}
$$

We now assume $p>0$ and

$$
\nu a_{\nu} \geqq-p, \quad \nu=1,2, \cdots, 2 n-1 ;
$$

then (4.1) gives

$$
\sum_{1}^{n}\left(\nu a_{\nu}+p\right) \leqq n\left\{\frac{4}{\pi}+p+\frac{p}{n} \sum_{n+1}^{2 n-1} \frac{2 n-\nu}{\nu}\right\},
$$

and, using Lemma 1 , we obtain $\sum_{1}^{n} \nu a_{\nu}<n\{4 / \pi+p(2 \log 2-1)\},(n \geqq 2)$. Also from (4.2) $\nu\left(\left|a_{\nu}\right|-a_{\nu}\right) \leqq 2 p, \quad(\nu=1,2, \cdots, 2 n-1)$; hence $\sum_{1}^{n} \nu\left|a_{\nu}\right|<n\{4 / \pi+p(1+2 \log 2)\},(n \geqq 1)$. In the same way the assumption $\nu b_{\nu} \geqq-p,(\nu=1,2, \cdots, 2 n-1)$, yields $\sum_{1}^{n} \nu\left|b_{\nu}\right|<n\{4 / \pi$ $+p(1+2 \log 2)\}$. A fortiori 


$$
\left|\sum_{1}^{n} \nu a_{\nu} \cos \nu x\right|<n\left\{\frac{4}{\pi}+p(1+2 \log 2)\right\}, \quad n \geqq 1,
$$

and

$$
\left|\sum_{1}^{n} \nu b_{\nu} \sin \nu x\right|<n\left\{\frac{4}{\pi}+p(1+2 \log 2)\right\}, \quad n \geqq 1 .
$$

Again from (4.1)

$$
\sum_{n+1}^{2 n-1}(2 n-\nu)\left(a_{\nu}+\frac{p}{\nu}\right) \leqq n\left(\frac{4}{\pi}+p\right)+p \sum_{n+1}^{2 n-1} \frac{2 n-\nu}{\nu}, \quad n \geqq 2 ;
$$

hence $\sum_{n+1}^{2 n-1}(2 n-\nu) a_{\nu} \leqq n(4 / \pi+p)$. But from (4.2) we have $(2 n-\nu)$ $\cdot\left(\left|a_{\nu}\right|-a_{\nu}\right) \leqq 2 p(2 n-\nu) / \nu ;$ hence

$$
\sum_{n+1}^{2 n-1}(2 n-\nu)\left|a_{\nu}\right| \leqq n\left(\frac{4}{\pi}+p\right)+2 p \sum_{n+1}^{2 n-1} \frac{2 n-\nu}{\nu} .
$$

Using again Lemma 1 , we get

$$
\sum_{n+1}^{2 n-1}(2 n-\nu)\left|a_{\nu}\right|<n\left\{\frac{4}{\pi}+p(4 \log 2-1)\right\},
$$

and similarly $\sum_{n+1}^{2 n-1}(2 n-\nu)\left|b_{\nu}\right|<n\{4 / \pi+p(4 \log 2-1)\}$. Summarizing, we have the following theorem:

THEOREM 1. Suppose $|f(x)| \leqq 1$; if in addition

$$
\nu a_{\nu} \geqq-p, \quad \nu=1,2, \cdots, 2 n-1 ; p>0 ; n>0,
$$

then, for $\kappa=1, \cdots, n$,

$$
\begin{array}{r}
\sum_{1}^{\kappa} \nu\left|a_{\nu}\right|<\kappa\left\{\frac{4}{\pi}+p(1+2 \log 2)\right\}, \\
\sum_{\nu=\kappa+1}^{2 \kappa}(2 \kappa-\nu)\left|a_{\nu}\right|<\kappa\left\{\frac{4}{\pi}+p(4 \log 2-1)\right\} .
\end{array}
$$

Similarly, if

then

$$
\nu b_{\nu} \geqq-p, \quad \nu=1, \cdots, 2 n-1 ; p>0 ; n>0
$$

$$
\begin{array}{r}
\sum_{1}^{\kappa} \nu\left|b_{\nu}\right|<\kappa\left\{\frac{4}{\pi}+p(1+2 \log 2)\right\}, \\
\sum_{\kappa+1}^{2 \kappa}(2 \kappa-\nu)\left|b_{\nu}\right|<\kappa\left\{\frac{4}{\pi}+p(4 \log 2-1)\right\} .
\end{array}
$$


For $\kappa=n=1$ we have also the inequalities $\left|a_{1}\right| \leqq 4 / \pi,\left|b_{1}\right| \leqq 4 / \pi$. Note that $4 / \pi=1.2732 \cdots, 1+2 \log 2=2.3862 \cdots, 4 \log 2-1=1.7725 \cdots$.

5. Estimates for partial sums. We now assume (4.7) and (4.10); applying Theorem 1, formula (2.2), and the inequality (2.7) we obtain

$$
\left|\frac{a_{0}}{2}+\sum_{1}^{\kappa} a_{\nu} \cos \nu x\right|<1+\frac{4}{\pi}+p(1+2 \log 2),
$$

for $\kappa=1, \cdots, n$, and

$$
\left|\sum_{1}^{\kappa} b_{\nu} \sin \nu x\right|<1+\frac{4}{\pi}+p(1+2 \log 2), \quad \kappa=1, \cdots, n .
$$

Using again (2.2) with $u_{\nu}=a_{\nu} \cos \nu x+b_{\nu} \sin \nu x$ we obtain $\left|s_{\kappa}(f ; x)\right|<1$ $+8 / \pi+2 p(1+2 \log 2),(\kappa=1, \cdots, n)$. Using now (1.8) and the assumption (1.4) we get

$$
\left|s_{\kappa}(f ; x)\right|<(1+8 / \pi) \mu+2 p(1+2 \log 2)<3.547 \mu+4.773 p .
$$

This is sharper than (1.6).

Note that the formula $s_{0}=a_{0} / 2=(2 \pi)^{-1} \int_{-\pi}^{\pi} f(x) d x$ gives $\left|s_{0}\right| \leqq \mu$, assuming (1.4) only. In a similar way formulas (2.3), (2.8), (4.9), and (4.12) give

$$
\begin{aligned}
&\left|\frac{a_{0}}{2}+\sum_{1}^{\kappa} a_{\nu} \cos \nu x\right|<1+\frac{6}{\pi}+p(4 \log 2-1), \\
&\left|\sum_{1}^{\kappa} b_{\nu} \sin \nu x\right|<1+\frac{6}{\pi}+p(4 \log 2-1), \\
&\left|s_{\kappa}(f ; x)\right|<1+\frac{10}{\pi}+2 p(4 \log 2-1), \\
& \kappa=1, \cdots, n .
\end{aligned}
$$

Since $1+10 / \pi=4.18309 \cdots, 8 \log 2-2=3.54518 \cdots$, this estimate again is sharper than (1.6).

Using again formula (1.8) and summarizing, we have the following theorem:

THEOREM 2. If $|f(x)| \leqq \mu$ and

$$
\nu a_{\nu} \geqq-p, \quad \nu b_{\nu} \geqq-p, \quad \nu=1, \cdots, 2 n-1 ; p>0,
$$

then for $\kappa=1, \cdots, n$

$$
\left|\frac{a_{0}}{2}+\sum_{1}^{\kappa}\left(a_{\nu} \cos \nu x+b_{\nu} \sin \nu x\right)\right|<\left\{\begin{array}{l}
(1+8 / \pi) \mu+2 p(1+2 \log 2) \\
(1+10 / \pi) \mu+2 p(4 \log 2-1) .
\end{array}\right.
$$


6. Estimates for $\sum_{\nu=l+1}^{2 l-1}(2 l-\nu)\left|a_{\nu+\lambda-1}\right|$ and related sums. In [9] the author proved, under the assumption (2.6), that

$$
\left|\sum_{\nu=1}^{l} \nu u_{\nu+\lambda-1}+\sum_{\nu=l+1}^{2 l}(2 l-\nu) u_{\nu+\lambda-1}\right| \leqq \frac{4}{\pi} l, \quad \lambda=1,2,3, \cdots ; l \geqq 1 ;
$$

here $u_{\nu}$ stands for $a_{\nu} \cos \nu x+b_{\nu} \sin \nu x$ or $b_{\nu} \cos \nu x-a_{\nu} \sin \nu x$. Hence, for $l>1, \sum_{\nu=1}^{l} \nu a_{\nu+\lambda-1}+\sum_{\nu=l+1}^{2 l-1}(2 l-\nu) a_{\nu+\lambda-1} \leqq 4 l / \pi$, and for $l=1,\left|u_{\lambda}\right| \leqq 4 / \pi$, $(\lambda=1,2,3, \cdots)$. Thus for $l>1$

$$
\begin{aligned}
\sum_{\nu=1}^{l} \nu\left(a_{\nu+\lambda-1}+\right. & \left.\frac{p}{\nu+\lambda-1}\right)+\sum_{\nu=l+1}^{2 l-1}(2 l-\nu)\left(a_{\nu+\lambda-1}+\frac{p}{\nu+\lambda-1}\right) \\
& \leqq \frac{4}{\pi} l+p\left\{\sum_{\nu=1}^{l} \frac{\nu}{\nu+\lambda-1}+\sum_{\nu=l+1}^{2 l-1} \frac{2 l-\nu}{\nu+\lambda-1}\right\} .
\end{aligned}
$$

We now assume for a given integer $N$ that

$$
\nu a_{\nu} \geqq-p, \quad \nu=1,2, \cdots, N,
$$

and suppose that

$$
2 l+\lambda-2 \leqq N
$$

then from (6.1), for $l>1$, we have

$$
\sum_{\nu=l+1}^{2 l-1}(2 l-\nu) a_{\nu+\lambda-1} \leqq \frac{4}{\pi} l+p \sum_{\nu=1}^{l} \frac{\nu}{\nu+\lambda-1} .
$$

Furthermore $\left|a_{\nu}\right|-a_{\nu} \leqq 2 p / \nu,(\nu=1, \cdots, N)$; hence using (6.4) we obtain

$$
\sum_{\nu=l+1}^{2 l-1}(2 l-\nu)\left|a_{\nu+\lambda-1}\right| \leqq \frac{4}{\pi} l+p\left\{2 \sum_{\nu=l+1}^{2 l-1} \frac{2 l-\nu}{\nu+\lambda-1}+\sum_{\nu=1}^{l} \frac{\nu}{\nu+\lambda-1}\right\} .
$$

Similarly, assuming

$$
\nu b_{\nu} \geqq-p, \nu=1, \cdots, N,
$$

we get, for $l>1$,

$$
\sum_{\nu=l+1}^{2 l-1}(2 l-\nu)\left|b_{\nu+\lambda-1}\right| \leqq \frac{4}{\pi} l+p\left\{2 \sum_{\nu=l+1}^{2 l-1} \frac{2 l-\nu}{\nu+\lambda-1}+\sum_{\nu=1}^{l} \frac{\nu}{\nu+\lambda-1}\right\} .
$$

A simple calculation yields

$$
\sum_{\nu=l+1}^{2 l-1} \frac{2 l-\nu}{\nu+\lambda-1} \leqq \frac{1}{l+\lambda} \sum_{\nu=l+1}^{2 l-1}(2 l-\nu)=\frac{l(l-1)}{2(l+\lambda)}
$$




$$
\sum_{\nu=1}^{l} \frac{\nu}{\nu+\lambda-1}<\frac{l^{2}}{l+\lambda-1} .
$$

We have thus proved the following theorem:

TheOREM 3. Under the assumptions (2.6), (6.2), (6.3), and (6.5) we have, for $l>1, \lambda=1,2,3, \cdots$,

$$
\sum_{\nu=l+1}^{2 l}(2 l-\nu) v_{\nu+\lambda-1}<\left\{\frac{4}{\pi}+p\left(\frac{l-1}{l+\lambda}+\frac{l}{l+\lambda-1}\right)\right\} l,
$$

where $v_{\nu}$ stands for $\left|a_{\nu}\right|$ or $\left|b_{\nu}\right|$.

Formula (6.6) remains true also for $l=1$.

7. Further estimates for partial sums. Assuming (2.6) we get, from (2.4) and (2.7),

$$
\left|U_{n}\right| \leqq 1+\frac{2 n}{\kappa}+\frac{1}{\kappa}\left|\sum_{\nu=n+1}^{n+\kappa}(n-\nu+\kappa) u_{\nu}\right|, \quad \kappa \geqq 1, n \geqq 1,
$$

where $u_{\nu}$ means $a_{\nu} \cos \nu x, b_{\nu} \sin \nu x$, or $a_{\nu} \cos \nu x+b_{\nu} \sin \nu x$. Replacing in (6.6) $l$ by $\kappa$ and $\lambda$ by $n-\kappa+1$, we get

$$
\sum_{\nu=l+1}^{2 l}(2 l-\nu) v_{\nu+\lambda-1}=\sum_{\nu=\kappa+1}^{2 \kappa}(2 \kappa-\nu) v_{\nu+n-\kappa} \equiv \sum_{\nu=n+1}^{n+\kappa}(n-\nu+\kappa) v_{\nu}
$$

hence, using (6.6) and (7.1), we obtain

$$
\left|U_{n}\right|<1+\frac{2 n}{\kappa}+\frac{4}{\pi}+p\left(\frac{\kappa-1}{n+1}+\frac{\kappa}{n}\right), \quad n \geqq \kappa \geqq 1 .
$$

The condition (6.3) becomes

$$
n+\kappa-1 \leqq N
$$

Here $U_{n}$ means $a_{0} / 2+\sum_{1}^{n} a_{\nu} \cos \nu x$ or $\sum_{1}^{n} b_{\nu} \sin \nu x$. We observe that $2 n / \kappa+p\{(\kappa-1) /(n+1)+\kappa / n\}$ decreases with increasing $\kappa$ where $\kappa(\kappa+1) \leqq 2 n^{2}(n+1) / p(2 n+1)$. We thus put

$$
\kappa=\left[\frac{1}{2}+\left\{\frac{1}{4}+\frac{2 n^{2}(n+1)}{p(2 n+1)}\right\}^{1 / 2}\right]
$$

obviously

$$
\kappa>-\frac{1}{2}+\left\{\frac{1}{4}+\frac{2 n^{2}(n+1)}{p(2 n+1)}\right\}^{1 / 2} \geqq 0 ;
$$

hence $\kappa \geqq 1$. We now get easily the relation 
and

$$
\begin{aligned}
\frac{2 n}{\kappa}+p \frac{\kappa}{n}< & \frac{2 n}{-\frac{1}{2}+\left\{\frac{1}{4}+\frac{2 n^{2}(n+1)}{p(2 n+1)}\right\}^{1 / 2}} \\
& +\frac{p}{n}\left(-\frac{1}{2}+\left\{\frac{1}{4}+\frac{2 n^{2}(n+1)}{p(2 n+1)}\right\}^{1 / 2}\right),
\end{aligned}
$$

$$
\frac{2 n}{\kappa}+p \frac{\kappa}{n}+p \frac{\kappa-1}{n+1}<\frac{2 p(2 n+1)}{n(n+1)}\left\{\frac{1}{4}+\frac{2 n^{2}(n+1)}{p(2 n+1)}\right\}^{1 / 2} \leqq 4 p^{1 / 2}
$$

if

$$
p \leqq 16 n / 9, \quad p \leqq 2 n^{4} /(2 n+1)^{2} .
$$

On putting $N=2 n-1$ in (7.3) we get $\kappa \leqq n$; in view of (7.4) this condition is satisfied if

$$
p \geqq \frac{2 n(n+1)}{(2 n+1)(n-1)},
$$

Thus with the restrictions (7.5) and (7.6) the assumptions (2.6) and (5.5) imply

$$
\left|U_{n}\right|<1+4 / \pi+4 p^{1 / 2} \text {. }
$$

In the cases $p>16 n / 9$ or $p>2 n^{4} /(2 n+1)^{2}$, (7.7) can be deduced from $a_{0}^{2} / 2+\sum_{1}^{n}\left(a_{\nu}{ }^{2}+b_{\nu}{ }^{2}\right) \leqq \pi^{-1} \int_{-\pi}^{\pi} f^{2}(x) d x \leqq 2$. Now

$$
\begin{aligned}
\frac{1}{2}\left|a_{0}\right|+\sum_{1}^{n}\left|a_{\nu}\right| & \leqq\left\{\frac{1}{2} a_{0}^{2}+\sum_{1}^{n} a_{\nu}^{2}\right\}^{1 / 2}\left(\frac{1}{2}+n\right)^{1 / 2} \\
& \leqq(1+2 n)^{1 / 2}<1+4 / \pi+4 p^{1 / 2}
\end{aligned}
$$

Finally in the case $p<2 n(n+1) /(2 n+1)(n-1)$ we can deduce $(7.7)$ from (2.5), (2.7), (4.8), and (4.11), which give $\left|U_{n}\right|<1+[n /(n+1)]$ $\cdot\{4 / \pi+p(1+2 \log 2)\}$ and this is in the present case less than $1+4 / \pi+4 p^{1 / 2}$ for $n>1$, since $[n /(n+1)] p^{1 / 2}<4 /(1+2 \log 2)$. Summarizing and using (1.8), we complete the proof of the following theorem :

THEOREM 4. Under the assumptions (1.4) and (5.5),

$$
\begin{aligned}
\left|\frac{a_{0}}{2}+\sum_{1}^{\kappa} a_{\nu} \cos \nu x\right| & <\left(1+\frac{4}{\pi}\right) \mu+4(p \mu)^{1 / 2}, \\
\left|\sum_{1}^{\kappa} b_{\nu} \sin \nu x\right| & <\left(1+\frac{4}{\pi}\right) \mu+4(p \mu)^{1 / 2}, \quad \kappa=1,2, \cdots, n .
\end{aligned}
$$


In the case $u_{\nu}=a_{\nu} \cos \nu x+b_{\nu} \sin \nu x$, we get from (7.1) and (6.6) in a similar way

$$
\left|U_{n}\right|<1+\frac{2 n}{\kappa}+\frac{8}{\pi}+2 p\left(\frac{\kappa-1}{n+1}+\frac{\kappa}{n}\right), \quad 1 \leqq \kappa \leqq n .
$$

We now put

$$
\kappa=\left[\frac{1}{2}+\left\{\frac{1}{4}+\frac{n^{2}(n+1)}{p(2 n+1)}\right\}^{1 / 2}\right]
$$

that is, in our previous argument $p$ is replaced by $2 p$. We get the following theorem:

TheOREM 5. Under the assumptions (1.4) and (5.5),

$$
\left|s_{\kappa}(f ; x)\right|<\left(1+\frac{8}{\pi}\right) \mu+4(2 p \mu)^{1 / 2}, \quad \kappa=1, \cdots, n .
$$

Since $1+8 / \pi=3.5464 \cdots<5$, and $4(2)^{1 / 2}<8$, our estimate is sharper than (1.7).

The results can be extended to almost periodic functions (for $p=0$ see [3], [7], [8], [9]) and to Fourier integrals; also the condition (1.3) can be generalized to "slow oscillation," improving upon some results of Fekete and the author.

\section{REFERENCES}

1. L. Fejér, On a theorem of Paley, this Bulletin, vol. 40 (1934), pp. 469-475.

2. M. Fekete, Proof of three propositions of Paley, ibid., vol. 41 (1935), pp. 138-144.

3. - Some generalizations of Paley's theorem on Fourier series with positive coefficients, Transactions of this Society, vol. 38 (1935), pp. 237-249.

4. O. Szász, Zur Konvergenztheorie der Fourierschen Reihen, Acta Mathematica, vol. 61 (1933), pp. 185-201.

5. ——, A Fourier-féle sor rêszletösszegeinck korlâtosságâról és összetartâsaról, Matematikai és Természettudományi Értesítö, vol. 50 (1933), pp. 125-146.

6. - Convergence properties of Fourier series, Transactions of this Society, vol. 37 (1935), pp. 483-500.

7. - On the partial sums of certain Fourier series, American Journal of Mathematics, vol. 59 (1937), pp. 696-708.

8. ——, Fourier-sorok részletösszegeiröl, Matematikai és Természettudományi Ertesítö, vol. 56 (1937), pp. 382-396.

9. - Some extremum problems in the theory of Fourier series, to appear in the American Journal of Mathematics.

University of Cincinnati 